\title{
VIRTUAL SIMULATION OF OVERTAKING MANEUVER OF AUTONOMOUS VEHICLE
}

\author{
MAGDOLEN Luboš ${ }^{*}$, DANKO Ján ${ }^{1}$, MILESICH Tomášś ${ }^{1}$, KEVICKÝ Igor ${ }^{1}$, \\ GALINSKÝ Marek ${ }^{2}$, BUCHA Jozef ${ }^{1}$, SKYRČÁK Robert ${ }^{1}$
}

\author{
${ }^{1}$ Slovak University of Technology in Bratislava, Faculty of Mechanical Engineering, Institute of Transport \\ Technology and Designing, Nám. slobody 17, 81231 Bratislava, Slovakia, e-mail: lubos.magdolen@stuba.sk \\ ${ }^{2}$ Slovak University of Technology in Bratislava, Faculty of Informatics and Information Technologies, Institute \\ of Computer Engineering and Applied Informatics, Ilkovičova 2, 84216 Bratislava 4
}

\begin{abstract}
The overtaking maneuver is an important maneuver for autonomous vehicles. It consists of overtaking another vehicle that is moving at a slower speed in the same lane. The article proposes an ahead maneuver on a straight road using software designed for PreScan simulations, while the obstacle is also a moving vehicle. The vehicle is equipped with technology-independent sensors (TIS) and camera.
\end{abstract}

KEYWORDS: Overtaking maneuver, Autonomous vehicle, Sensors

\section{Introduction}

Autonomous vehicles have been experiencing tremendous development recently. The goal of all the researchers involved in their design is to bring a vehicle that is safe on the road and at the same time comfortable and economical to operate. The driver had to deal with tasks and decisions related to driving in non-autonomous vehicles. For autonomous vehicles, the computer will have to decide. However, there are many different driving maneuvers for which such an artificial intelligence computer must be prepared. It is therefore up to researchers to define the problems that autonomous vehicles may encounter and to find solutions to such problems.

This paper is about simulating an overtaking maneuver in a PreScan environment. This software is a simulation platform for the development of ADAS (Advanced Driver Assistance Systems) and provides the ability to simulate maneuvers that involve several elements, such as infrastructure, vehicles, sensors but at the same time it is possible to simulate traffic, buildings, weather. This software is connected to the MATLAB / Simulink software, while the Simulink session is a slave to the Prescan simulation core.

The vehicle performs an overtaking maneuver in the simulation. To achieve this, it is necessary to coordinate several aspects, and thus control the longitudinal and lateral dynamics (steering, acceleration, braking) with the support of monitoring their surroundings using sensors. The vehicle must interact with its urban environment and its goal is to adjust its preselected trajectory to avoid an obstacle. The aim of this study is to gather all the necessary components to perform an overtaking maneuver in the PreScan simulation software.

\section{Modeling of the overtaking maneuver}

PreScan software was used to simulate the overtaking maneuver. This software allows the simulation of Advanced Driver Assistance Systems (ADAS). This technology consists of 
various systems ensuring road safety, in our case, for example, the detection of objects while driving. One of the ADAS applications used is Advanced cruise control (ACC), which is particularly helpful on the highway, where drivers can find it difficult to monitor their speed and other cars over a long period of time can automatically accelerate, slow down, and at times stop the vehicle, depending on the action other objects in the immediate area.

The simulation includes a dual carriageway and two vehicles. The first vehicle (A) represents a moving obstacle, and the second vehicle (B) tries to perform an overtaking maneuver. The aim of the simulation is for vehicle $B$ to overtake vehicle $A$.

\subsection{Build a simulation}

There are several steps you need to take to perform the simulation. Each step also contains a description of the characteristics that the scenario must meet to simulate an overtaking maneuver.

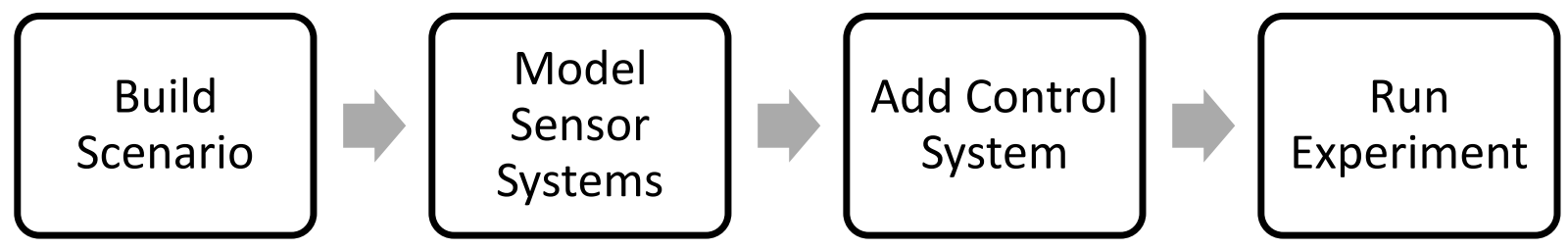

Fig. 1: Graphic illustration of the 4 steps for simulation in the PreScan software

Fig graphically shows the given steps. Before performing a simulation in PreScan, users should follow some recommendations:

1. The creation of the scenario should follow the numerical order of execution (Figure 1).

2. Make a comparison between the elements that make up the scenario and the simulation conditions specified by the user.

3. Finally, check and verify the elements used in the scenario to avoid incorrect information or data during the simulation.

The following part of the chapter explains the 4 steps of the simulation process when modeling the overtaking maneuver.

\subsection{Build scenario}

In the first step, it is necessary to create an environment in which the simulation will take place. PreScan provides the creation of a virtual world. In this step, you need to create a road and place vehicles on it.

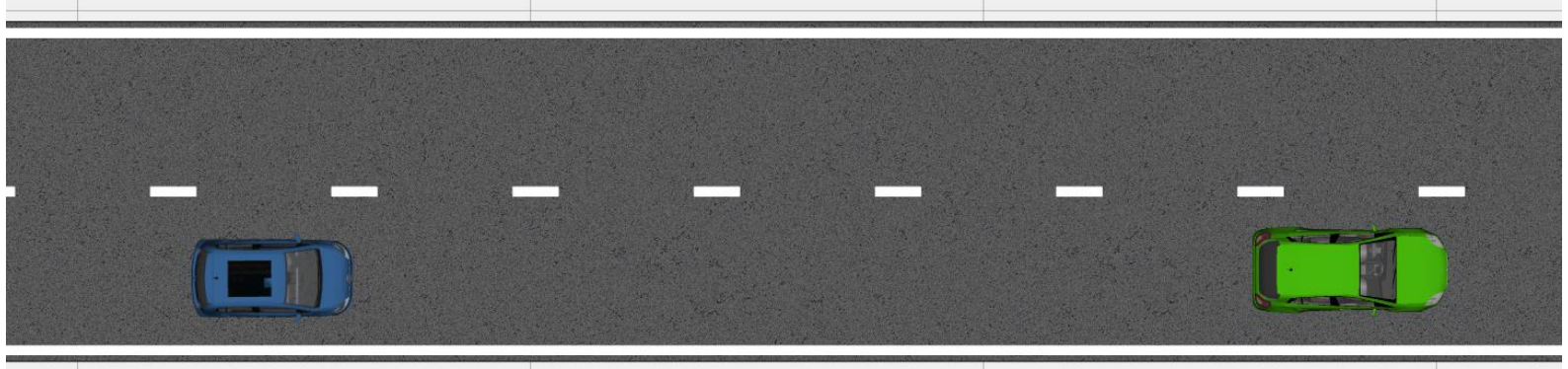

Fig. 2 Building a scenario

The scenario in detail consists of a two-lane carriageway $2500 \mathrm{~m}$ long with the 3,5 m width of one lane. Each lane of the scenario is designated with a name, which is: left lane (1) and right lane (2). Part of the scenario are also vehicles, referred to in the program as Actors located in 
the right lane of the highway. For the simulation, Fiat Bravo Hatchback vehicle was chosen as the overtaking vehicle (vehicle A) and Volkswagen UP as the vehicle performing the overtaking maneuver (vehicle B). The vehicles are separated by a distance that allows an overtaking maneuver.

The vehicles used in the simulation had to be modeled in the subprogram Model Preparation Tool which belongs to PreScan. It is necessary to enter their basic dimensional, dynamic, kinematic properties and data on the engine, transmission, and steering. A CAD model of the vehicle can also be used for visualization.

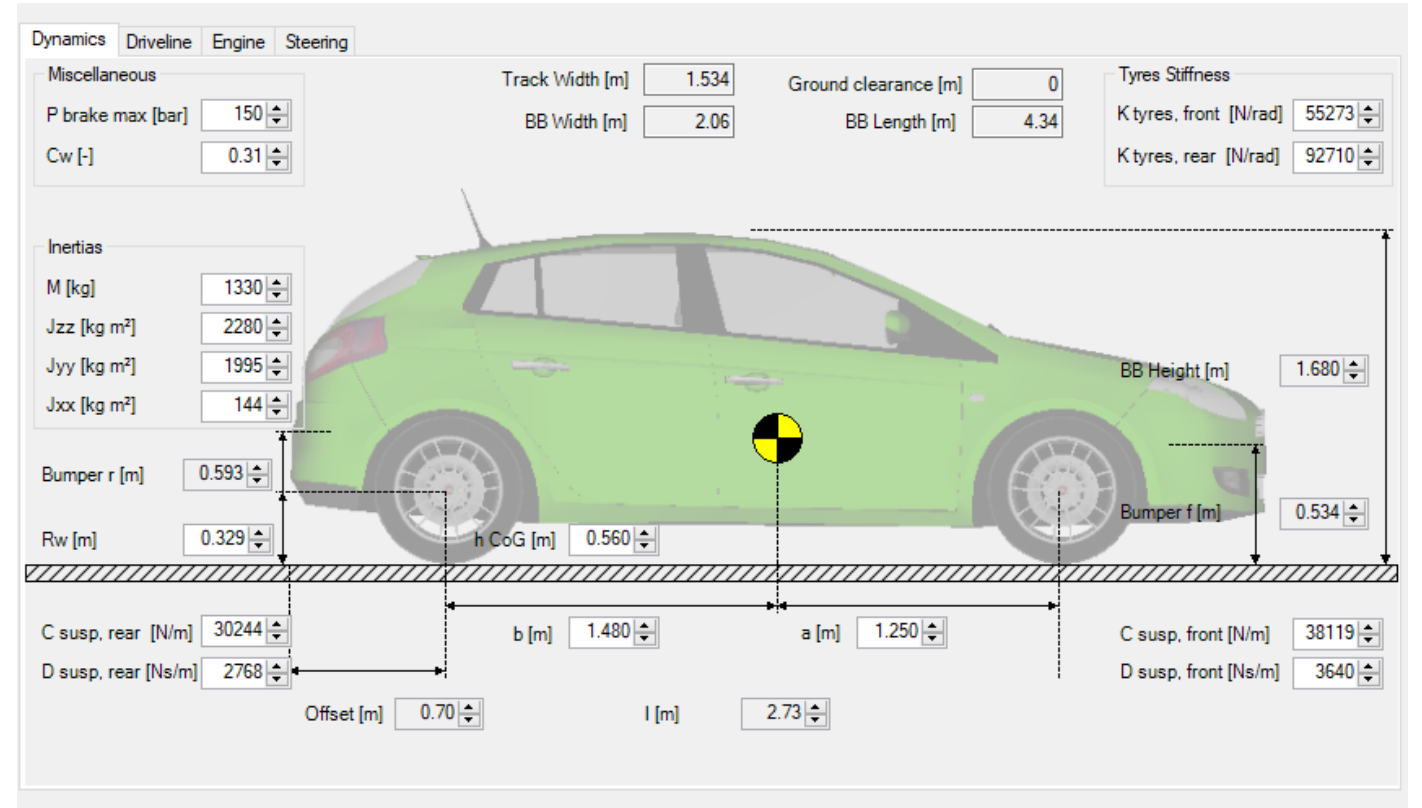

Fig. 3 Dynamics properties of an actor

\subsection{Model of sensor system}

The next step during the creation of the simulation is to equip the vehicles with sensors. In the case of an overtaking maneuver, it is not necessary for the sensors to be on the lead vehicle (A), but they must certainly be on the vehicle (B), for this reason only the Volkswagen UP (B) was equipped with sensors for simulation purposes.

Simulation software provides a plenty of sensors to choose from that we know from real practice. There are cameras, Lidars, Radars, Ultrasonic sensors to choose from. A TIS (Technology Independent Sensor) and camera was chosen for the simulation.

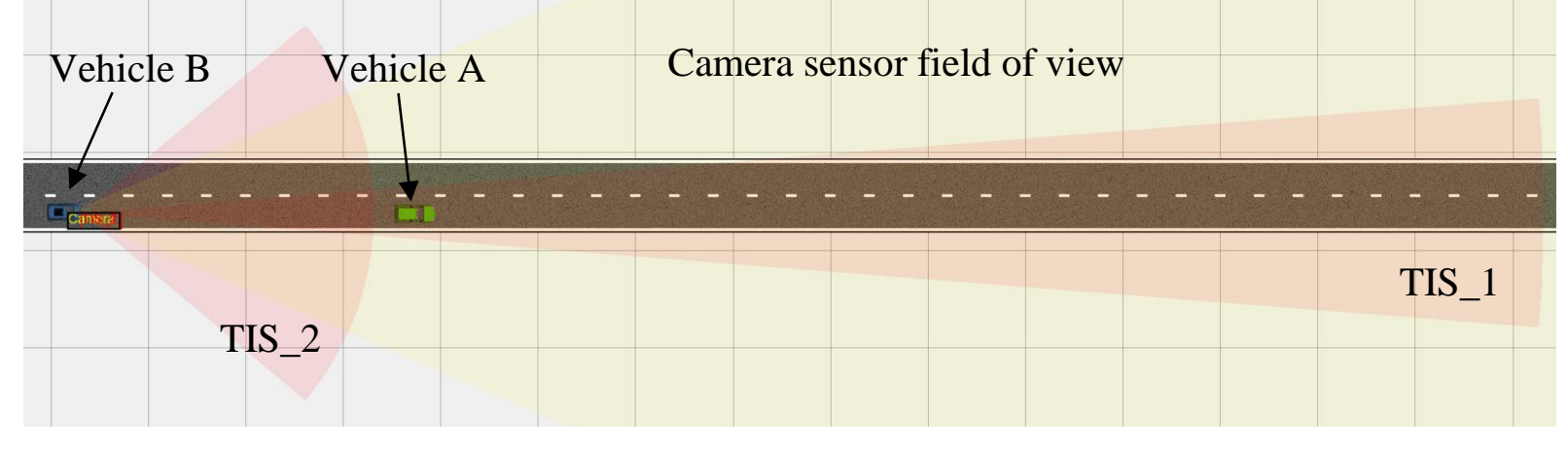

Fig. 4 Vehicles with sensors (range on)

There are 2 Independent Technology Sensors (TIS) on the vehicle. They are located front of the vehicle in the bumper. In this case, TIS_1 has the parameters Beam range $150 \mathrm{~m}$, Beam 
angle $9^{\circ}$, while TIS_2 Beam range $30 \mathrm{~m}$, Beam angle $80^{\circ}$ The camera sensor have resolution of $800 \times 600$ pixels with focal length of $7.5 \mathrm{~mm}$.

The TIS helps the user to increase his general knowledge and understanding of an active scanning sensor. Its operation is not tied up to a specific technology (such as that for radar, lidar or laser scanner), but the TIS does work according to common operating principles seen with these devices.

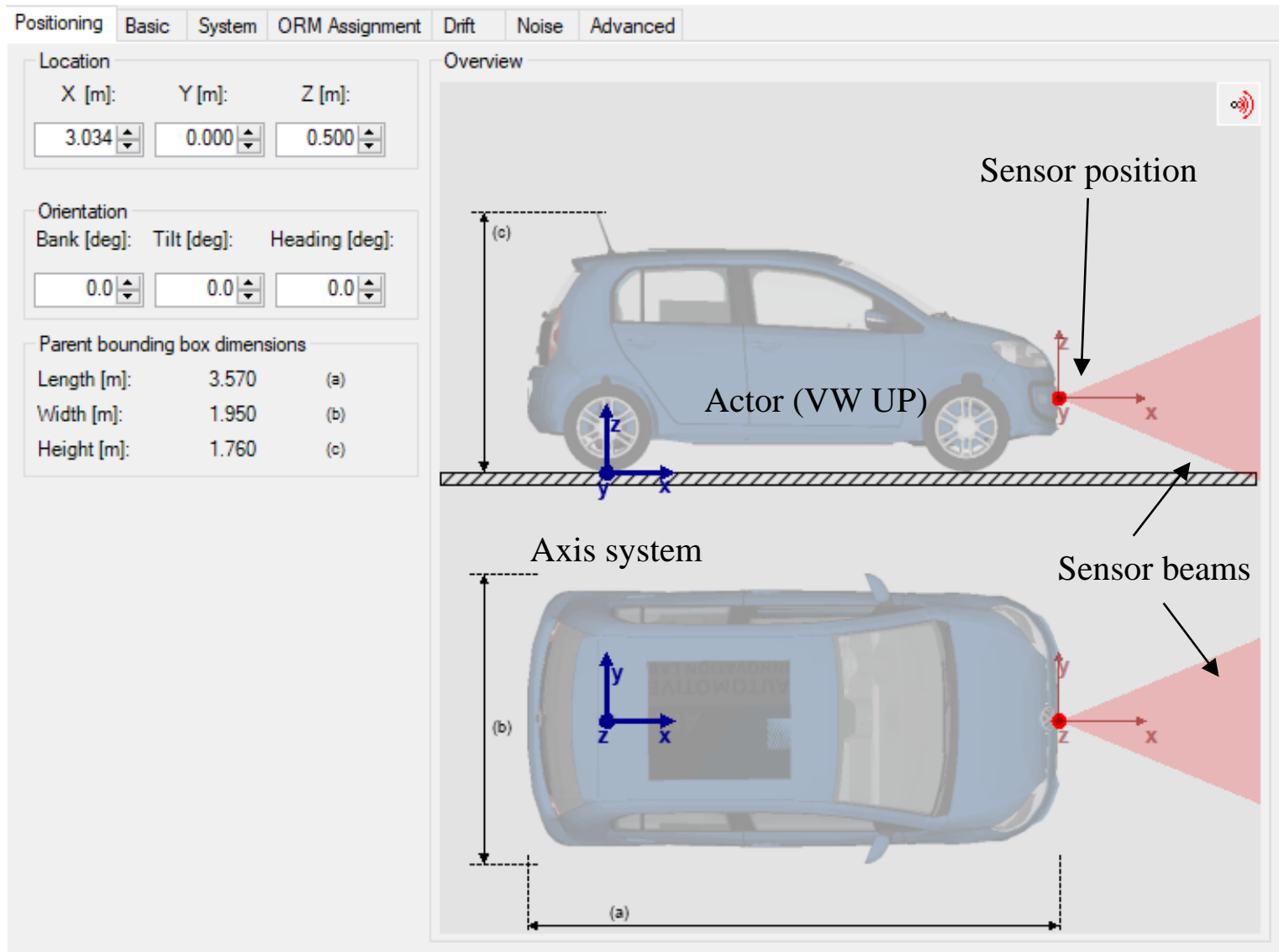

Figure 1: Configuration of sensor on vehicle (B)

\subsection{Add control system}

Control systems should be understood broader than traditional automotive control systems such as ESP and ACC. Control systems also include object segmentation algorithms, sensor fusion algorithms, decision making algorithms, etcetera.

This step no longer takes place in PreScan, but in MATLAB / Simulink. After completing the previous two steps, you can run the experiment analysis. During the experiment analysis in PreScan, the experiment configuration is checked. In particular, the consistency of the simulation speed is checked in the experiment. In the event of a fault, the user can enter new values for the identified problem areas. After successfully reviewing all steps of the analysis, the user may decide to set up an experiment. Building involves generating all the files needed to run the experiment. After creating the experiment, it is possible to generate a compilation sheet (Simulink representation of the experiment). The compilation list contains information on the tracking of vehicles, infrastructure, sensors, and display ports used in the scenario performed in the PreScan program. In addition, the compilation list contains all relevant connections to the PreScan simulation engine and existing actors in the various PreScan classes. 
In Simulink it is necessary to create a string of functions that can control commands from the inputs that it has available. There are two vehicles in the simulation. The vehicle (A) it represents to be overtaken has a simple control generated by the PreScan program. This vehicle follows a predefined trajectory and tries to secure a track according to it. The vehicle considers its dynamic properties, especially in the control of the vehicle in the transverse direction, but in this simulation, where the vehicles follow the same road, this steering component does not occur.

The vehicle (B) is based on the same steering model, i.e., it follows a predefined trajectory as a priority. It moves at a higher speed than the vehicle (A), so it catches up with it after a short time. use ACC to adjust your speed to be able to replace the distance behind the vehicle (A). The vehicle (B) evaluates that the vehicle (A) is moving at a lower speed, and therefore that it must overtake it if it wants to maintain its preselected desired speed. Trajectory change and overtaking the vehicle (A) Inputs that drive the vehicle are data from TIS sensors and camera about the position of the vehicle in front of it.

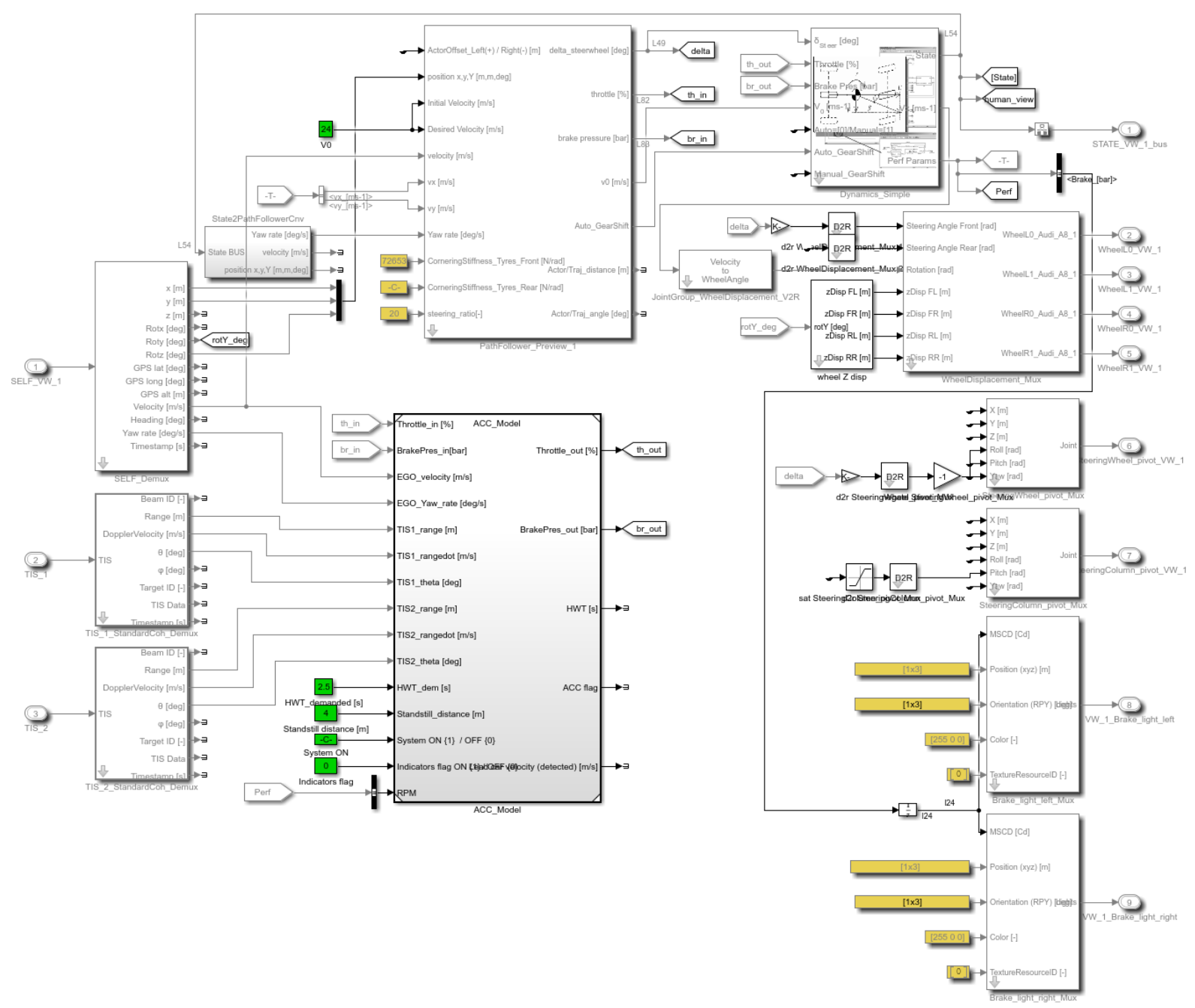

Fig. 6 Control system realisation for co-simulation

As already mentioned, the vehicle control commands are based on Simulink. The function blocks controlling the vehicle (B) can be seen at Figure 6. 


\subsection{Run experiment}

The experiment can be run when the scenario contains all the actors, infrastructure, and sensors that the simulation requires, and when a compilation sheet has been developed. PreScan provides the ability to run a visualization of such a simulation through another window called VisViewer. Here, a complete 3D view of the created virtual world is possible, while it is possible to set viewpoints, for example as a default view from above or a view from the vehicle.

\section{Lane change}

If the vehicle evaluates that it is going to perform a preliminary maneuver, it will start planning to change its trajectory. The change of this trajectory is based on the calculation according to the captured data from the sensors and its shape is determined according to predefined parameters. The vehicle moves along a pre-planned trajectory until it evaluates that it is going to perform a preliminary maneuver. It then calculates a new trajectory, overtakes the obstacle, and continues a straight path in the new lane. After overcoming the obstacle and reaching a safe distance, the vehicle calculates another trajectory change to return to the original lane and then tracks the original trajectory again.

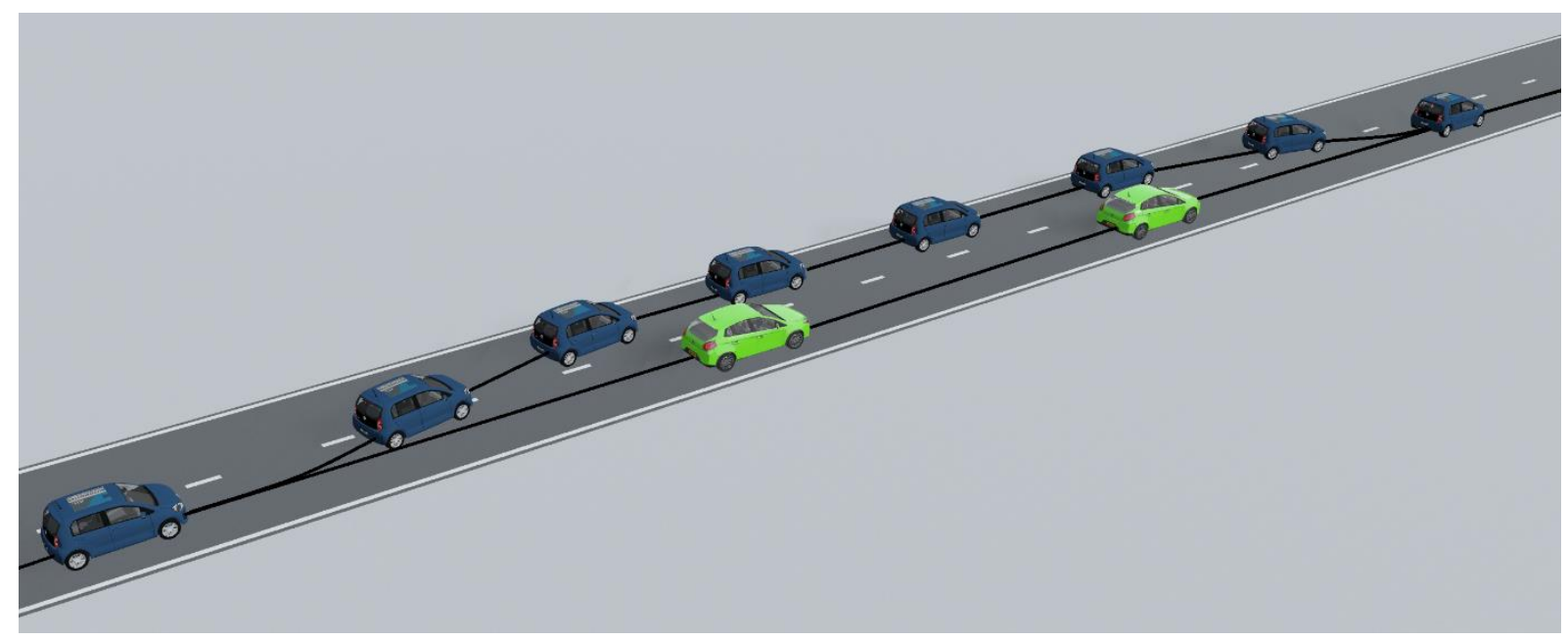

Fig. 7 3D view in VisViewer to simulation

Driving is based on the Stanley method. In this method, the input to the function for calculating the angle of rotation of the front wheels is the deviation of the distance between the center of the front wheels and the nearest point of the desired trajectory. The desired trajectory must therefore be planned for the center of the front wheels of the vehicle.

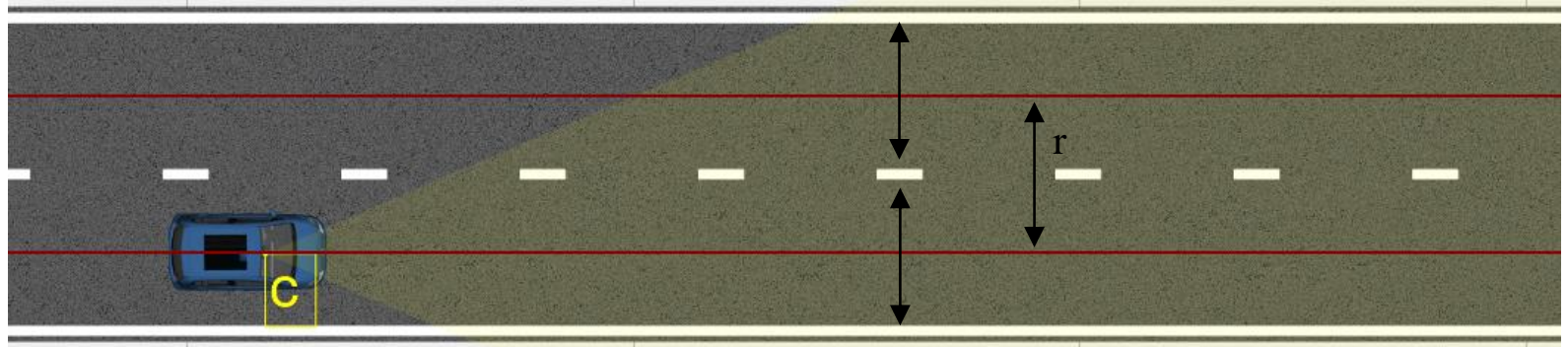

Fig. 8 Capturing the road with camera sensor

The vehicle captures the road ahead with the front camera. From the obtained data he can calculate the width of the lane in which he moves and the width of the second lane (the lane he 
gets in case of the overtaking maneuver). The vehicle calculates the position of the center of both lanes and the distance between these centers. Let us denote this distance as (r).

Through the sensors, the vehicle knows the distance (x) from the slower vehicle in front of it, as well as the dimensions of its rear part (a). To these dimensions, it adds a safe boundary (b) which creates a space around the overtaking vehicle into which no part of the overtaking vehicle may enter. (Figure 9)

Based on the lane center distance $r$, the program calculates the center of turning circles (C) according to the Ackermann steering geometry. Let's mark the center of the front wheels as $(\mathrm{F})$. At this point, a perpendicular line ( $\mathrm{p}$ ) is guided with respect to the direction of movement of the vehicle. On the perpendicular line, a point $(\mathrm{M})$ is formed at a distance $\left(\mathrm{r}_{\mathrm{f}}\right)$ from point $(\mathrm{F})$. The radius $r_{f}$ is known from the calculation of the hypotenuse from a right triangle, where $(r)$ is the distance between the centers of the lanes and $(\mathrm{w})$ is the wheelbase of our vehicle. The program then inserts a circle with radius $\left(\mathrm{r}_{\mathrm{f}}\right)$ at point $(\mathrm{M})$. This creates a circle $\left(\mathrm{c}_{1}\right)$.

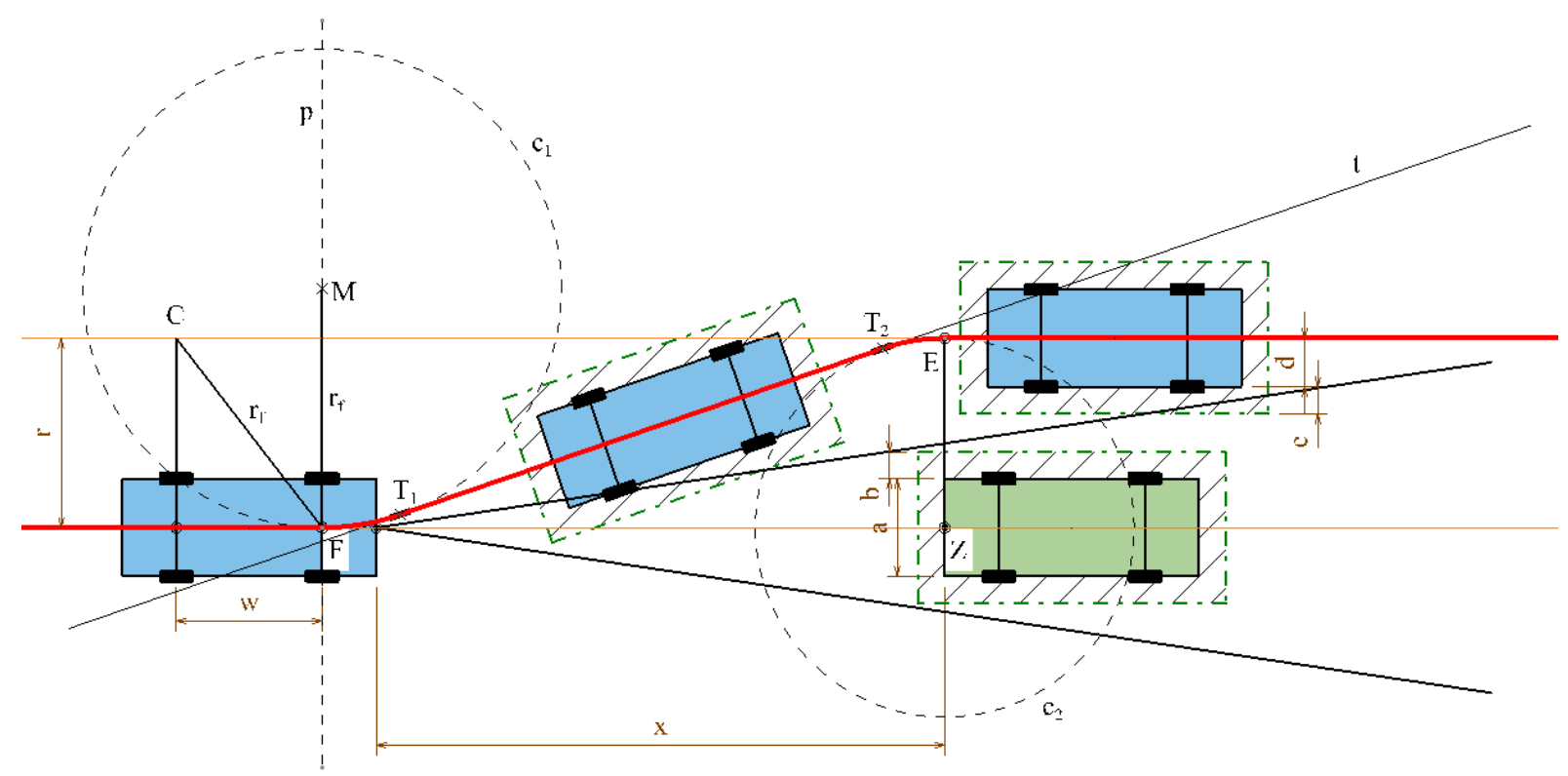

Fig. 9 Scheme of defining a new trajectory

The circle $\left(\mathrm{c}_{2}\right)$ has a center in the middle of the back of the obstacle $(\mathrm{Z})$. Its radius is the calculated perpendicular distance between the point $(Z)$ and the center of the second lane. During the calculation, a check of the minimum distance is made to see if the vehicle can be circled, i.e., whether the circled vehicle is not very close to the second lane. The radius of the circle must be greater than the sum of the perpendicular length of the obstacle a / 2, the safe limit (b), half the width of the overtaking vehicle (d) and the safe limit (e). If this condition is not met, no overtaking maneuver will take place.

If both circles have been created and the minimum distance condition has been met, the program creates a tangent to both circles $(\mathrm{t})$. The new trajectory is then part of the arc of the circle $\left(c_{1}\right)$ from point $(F)$ to point $\left(T_{1}\right)$, part of the tangent from point $\left(T_{1}\right)$ to point $\left(T_{2}\right)$ and part of the arc of the circle $\left(\mathrm{c}_{2}\right)$ from point $\left(\mathrm{T}_{2}\right)$ to point $(\mathrm{E})$.

The vehicle stops moving along the predetermined trajectory and starts tracking its planned new trajectory. The vehicle (B) continues to monitor the vehicle (A) via sensors and evaluates its safe detour.

When the vehicle overtakes an obstacle, it starts planning a trajectory to return to the original lane. The camera constantly monitors the area in front of it and constantly evaluates the position 
of the lane centers. The program to the center of the front wheels $(\mathrm{F})$ guides the perpendicular ( $\left.\mathrm{p}_{1}\right)$ with respect to the direction of travel. A point $\left(\mathrm{M}_{3}\right)$ is formed on the perpendicular at a distance $(r)$ from point $(F)$. This point is the center of a circle $\left(c_{3}\right)$ which has a radius $(r)$. The program also creates a point $\left(\mathrm{M}_{4}\right)$ in the middle of the lane where the vehicle moves at a distance $\left(\mathrm{x}_{\mathrm{d}}\right)$ from the center of the front wheels $(\mathrm{F})$. The program creates a circle centered at a point $\left(\mathrm{M}_{4}\right)$ with a radius ( $\left.\mathrm{r}\right)$ at that point. The program then creates a tangent to both circles $\left(\mathrm{t}_{2}\right)$. The new trajectory is then part of the arc of the circle $\left(\mathrm{c}_{3}\right)$ from point $(\mathrm{F})$ to point $\left(\mathrm{T}_{3}\right)$, part of the tangent from point $\left(\mathrm{T}_{3}\right)$ to point $\left(\mathrm{T}_{4}\right)$ and part of the arc of the circle $\left(\mathrm{c}_{4}\right)$ from point $\left(\mathrm{T}_{4}\right)$ to point (P). The vehicle then follows the created new trajectory, and after passing it, it continues to follow the original trajectory in the original lane. Figure10.

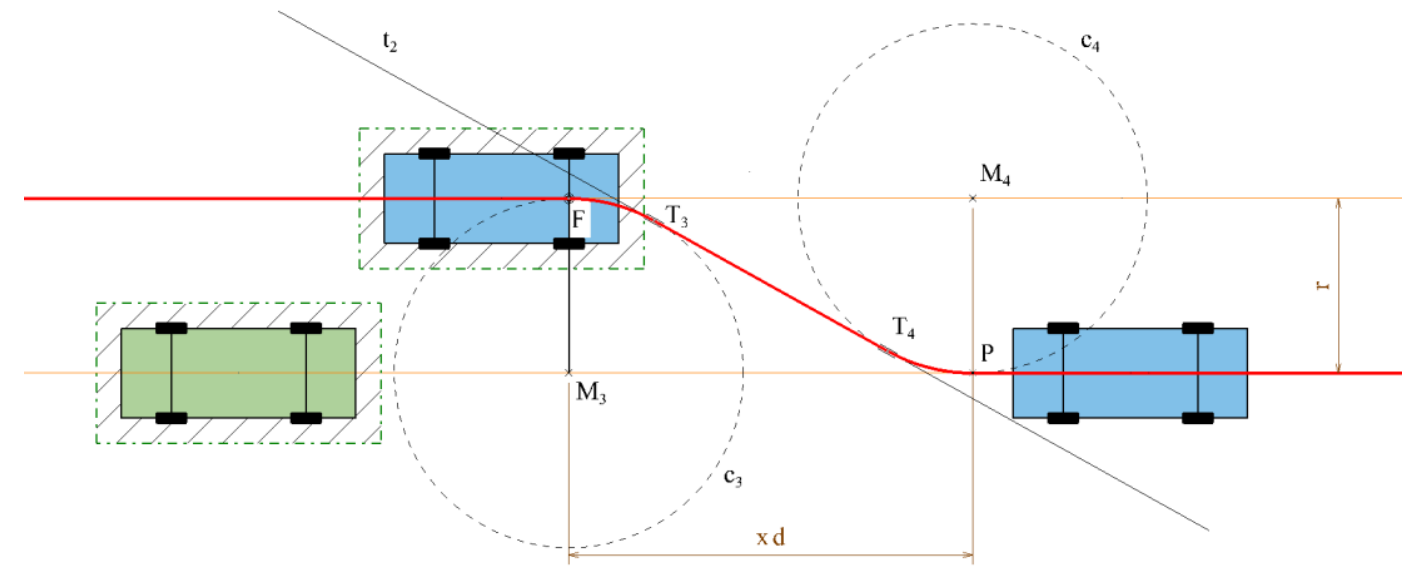

Fig. 10 Scheme of return to the original lane

\subsection{Simulation conditions}

To fulfill a defined driving maneuver (to overtake the vehicle), the simulation must meet the following requirements:

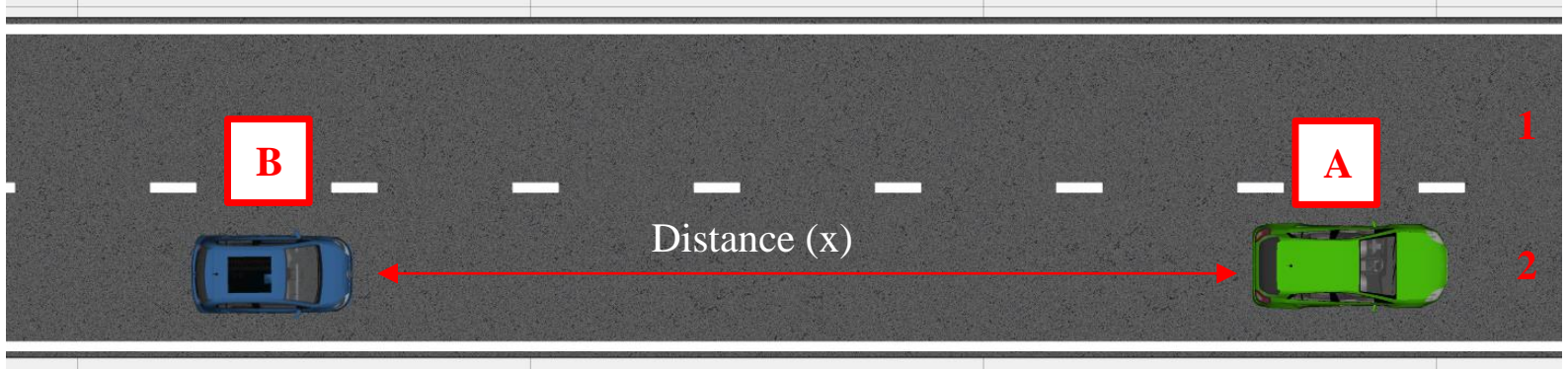

Fig. 11 Position of vehicles

- Vehicles (A) and (B) are separated by a sufficient distance (x)

- Vehicle (B) is faster than vehicle (A)

- Vehicles (A) and (B) are in the same lane (2) and the vehicle (B) must enter the side lane (1) when overtaking

- Vehicle (A) must go approximately in the middle of the lane (2)

The result is then that the vehicle (B) achieves the overtaking maneuver to the vehicle (C).

\section{Results}

The overtaking maneuver is divided into three phases, which are:

1. Deviation from the lane 
2. Driving in a lane, overtaking a vehicle

3. Return to the original lane.

In the first phase, the vehicle (B) starts to move from the right lane (2) to the left lane (1). In the second phase of the maneuver, the vehicle (B) moves in the lane (1) until the vehicle (A) circulates. Finally, in phase 3, the vehicle (B) returns to the original lane (1). The following sections describe how the vehicle (B) performs the overtaking maneuver.

\subsection{Deviation from the lane}

At this stage of the maneuver, the vehicle (B) should have a sufficient distance (x) from the vehicle (A) to prevent a collision. The vehicle can reduce its speed via the Adaptive Cruise Control system via the input from the TIS sensors to achieve the safe distance required for the overtaking maneuver.

\subsection{Driving in the lane, overtaking the vehicle}

During the lane driving phase, the vehicle detects whether there is a risk of a collision via the TIS sensor. If it is safe, the vehicle (B) will accelerate until it overtakes the vehicle (A). The vehicle (B) continues to drive in lane (1) and the vehicle (A) continues to drive in lane (2).

\subsection{Return to the original lane}

In the last phase, the vehicle (B) returns to lane (2) when it reaches a safe distance from the vehicle (A) and thus completes the overtaking maneuver. Both vehicles continue to drive in a straight line.

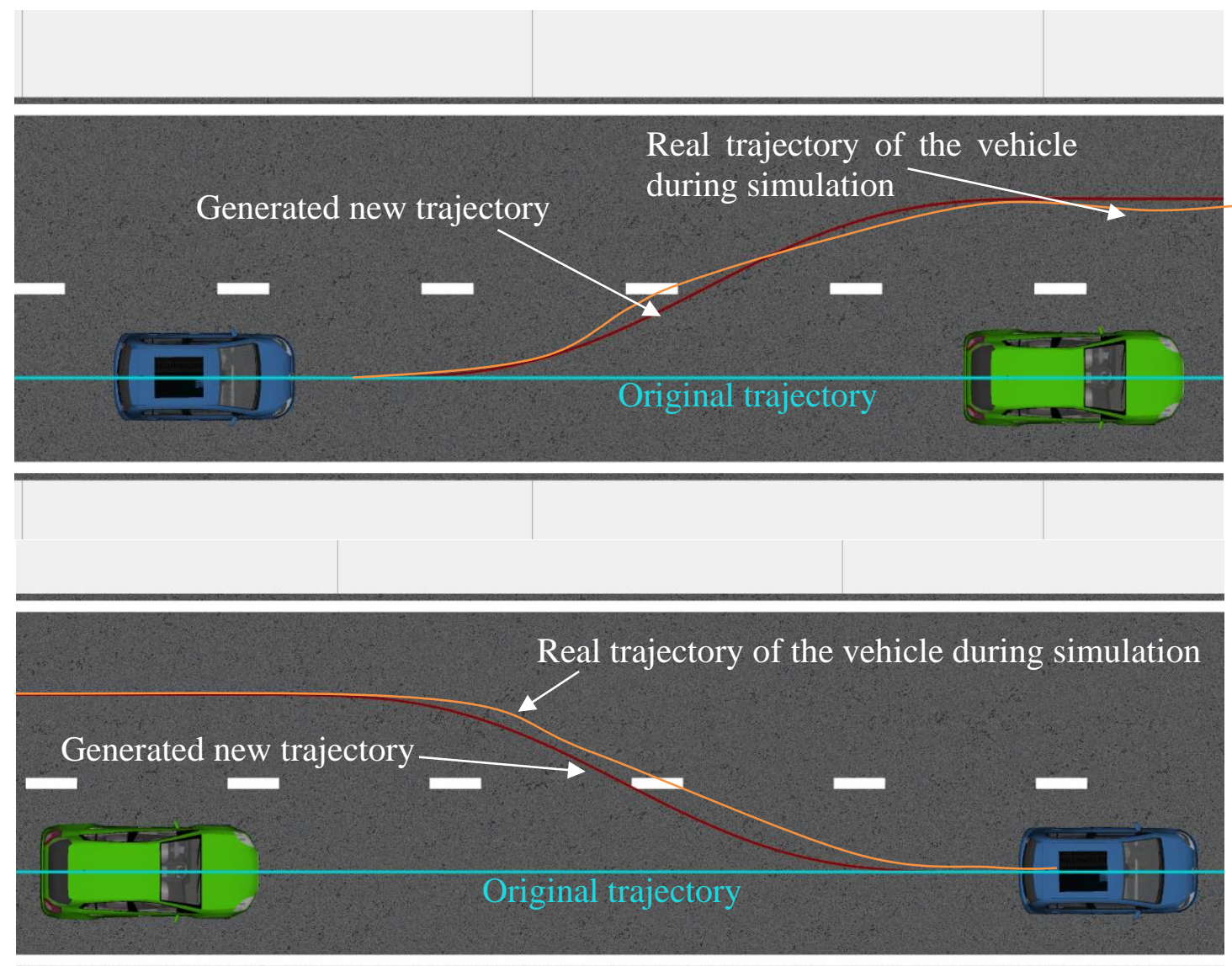

Fig. 12 Process of overtaking manoeuvre 


\section{CONCLUSION}

Modes of transport are constantly evolving, and autonomous vehicles will play a major role in transport in the future. New technologies are needed to ensure that autonomous vehicles are safe and able to perform the various maneuvers needed for smooth traffic. The proposed maneuver control must always be tested. Physical verification of the proposed model is very expensive, so there are various simulation software designed for such verification in the virtual world. One such software is PreScan, which has also been used in this work. Because PreScan contains various libraries, such as vehicles (called Actors in software), road infrastructure, sensors, weather conditions, it is possible to create driving maneuver scenarios for Autonomous Vehicles without the need to have them physically.

The aim of the research was to design a model of a safe overtaking maneuver without collisions between vehicles. The benefit of this work is to increase the understanding of the overtaking maneuver using simulation software.

\section{ACKNOWLEDGEMENT}

This work was supported by the Slovak Research and Development Agency under Contract No. APVV-19-0406.

\section{REFERENCES}

[1] Wang, C.S., Liu, D.Y., Hsu, K.S. "Simulation and application of cooperative driving sense systems using prescan software”, Microsyst. Technol., 2018.

[2] Snider, J. "Automatic Steering Methods for Autonomous Automobile Path Tracking", Pennsylania, Carnegie Mellon University, 2009. CMU-RI-TR-09-08.

[3] Zhang, M., Zhang, T., Zhang, Q. “An autonomous overtaking maneuver based on relative position information”, in: Proceedings of IEEE Vehicular Technology Conference, pp. 1 $-6,2018$.

[4] Chen, X., Miao, Y., Jin, M., Zhang, Q. "Driving decision-making analysis of lanechanging for autonomous vehicle under complex urban environment", in: Proceedings of the 29th Chinese Control and Decision Conference, CCDC, 2017, pp. 6878 - 6883, 2017.

[5] Milanés, V., Llorca D. F., Villagrá, J., Pérez, J., Fernández, C., Parra I., et al. "Intelligent automatic overtaking system using vision for vehicle detection”, Expert Syst. Appl. 39 pp. $3362-3373,2012$.

[6] Easa, S. M., Diachuk, M. "Optimal speed plan for the overtaking of autonomous vehicles on two-lane highways", Infrastructures 5, 2020. 